\title{
Estudio de factores asociados con amputación, en pacientes diabéticos con ulceración en pie
}

\author{
J.T. REAL COLLADO, M. VALLS, M.L. BASANTA ALARIO*, F.J. AMPUDIA BLAS- \\ CO, J.F. ASCASO GIMILIO, R. CARMENA RODRÍGUEZ
}

Unidad de Referencia de Diabetes, Servicio de Endocrinología y Nutrición. Hospital Clí nico Universitario. Departamento de Medicina. Universidad de Valencia. *Escuela Uni versitaria de Fisioterapia. Valencia.

\author{
STUDY OF FACTORS ASSOCIATED WITH AMPUTATION IN DIABE - \\ TIC PATIENTS WITH FOOT ULCERATION
}

\begin{abstract}
RESUMEN
Objetivo: Analizar factores asociados con amputación en pacientes diabéticos que presentan úlceras en sus pies, con el fin de identificar sujetos con alto riesgo para amputación.

Método: Hemos estudiado 152 diabéticos que acudieron a la Unidad del pie diabético desde enero de 1996 hasta junio de 1998 con úlceraciones en sus pies. En 14 de ellos fue necesaria la amputación. Se excluyeron sujetos con gangrena.

Resultados: Los factores asociados con amputación fueron: antecedentes personales de amputación previa (odds ratio 3,7; 1,01-13,7), retinopatía proliferativa, osteomielitis y, de forma independiente, la presencia de vasculopatía $(7,1 ; 1,88-27,2)$ e infección grave $(14,4 ; 2,92-71,2)$.

Conclusión: Los pacientes diabéticos con úlceras en pie que presenten antecedentes de amputación, signos clínicos de vasculopatía periférica, infección grave, osteomielitis o retinopatía avanzada son de alto riesgo para la amputación y en ellos se deben instaurar precozmente medidas terapéuticas y preventivas intensas.
\end{abstract}

PALABRAS CLAVE: Pie diabético. Amputación no traumática. Factores de riesgo para amputación. Úlceras del pie diabético.

\begin{abstract}
Objective: To analyse risk factors for amputation in diabetic foot ulcers.

Method: We have studied 152 diabetic patients (in 14 food ulcers treatment was amputation) who were attendance between January 1996 and June 1998 in the diabetic foot Unit. Subjects with gangrene were excluded.

Results: Risk factors for amputation were: previous history of amputation (odds ratio 3.7; 1.0113.7), proliferative retinopathy, osteomielitis, and independently clinical signs of peripheral vasculopathy $(7.1 ; 1.88$ 27.2) and severe infection $(14.4 ; 2.92-71.2)$.

Conclusion: Diabetic subjects with foot ulcers and previous history of amputation, proliferative retinopathy, osteomielitis, clinical signs of peripheral vasculopathy and/or severe infection were a high risk group for amputation and in this group aggressive therapeutical and preventive approaches should be done in order to prevent amputation.
\end{abstract}

KEY WORDS: Diabetic foot ulcers. Risk factors for amputation. Non traumatic amputation.

Real Collado JT, Valls M, Basanta Alario ML, Ampudia Blasco FJ, Ascaso Gimilio JF, Carmena Rodríguez R. Estudio de factores asociados con amputación en pacientes diabéticos con ulceración en pie. An Med Interna (Madrid) 2001; 18: 59-62.

\section{INTRODUCCIÓN}

La diabetes mellitus (DM) es un importante problema de Salud Pública por su elevada prevalencia y morbimortalidad (1). En los Estados Unidos representa la mayor causa de morbilidad, mortalidad y de gasto económico (1-5). Los pacientes diabéticos tienen un elevado riesgo de padecer complicaciones crónicas cardiovasculares, renales, oftalmológicas, neurológicas y podológicas cuya incidencia crece anualmente (6-9). En el año 1994 el coste directo por diabetes en España fue de 90.000 millones de pesetas siendo la hospitalización el 58\% del total del coste (10). El 15\% de los pacientes diabéticos desarro- llarán a lo largo de su evolución lesiones en sus pies (11). Se calcula que la prevalencia de las alteraciones de pie en DM está en torno al $10 \%$ (12), afectando con mayor frecuencia a la población de diabéticos, entre 45-65 años. Estas alteraciones pueden, en su evolución, requerir la amputación, lo que supone un elevado impacto personal, social, laboral y económico. Los sujetos con DM tienen 10 veces más posibilidades de sufrir una amputación no traumática que la población homóloga no DM, siendo la mortalidad perioperatoria del $6 \%$ y la postoperatoria de hasta un $50 \%$ a los 3 años (11-13). La implantación de programas de prevención y tratamiento de la ulceración en el pie diabético consigue reducir la tasa de amputaciones.

Trabajo aceptado: 3 de Julio de 2000

Correspondencia: J.T. Real. Servicio de Endocrinología y Nutrición. Hospital Clínico Universitario de Valencia. Avda. V. Blásco Ibáñez 17 - 46010 Valencia. 
El objetivo de nuestro estudio es conocer y analizar los factores asociados con amputación no traumática (AMP) en pacientes diabéticos con ulceración en pie atendidos en la Unidad de Pie diabético, con el fin de identificar a los sujetos de alto riesgo de amputación.

\section{MATERIAL Y MÉTODOS}

\section{SUJETOS}

Hemos estudiado 152 pacientes con el diagnóstico de Diabetes Mellitus que acudieron de forma consecutiva a la Unidad de Pie diabético desde Enero de 1996 hasta Junio de 1998 con pies de riesgo grado 2 y 3 según la clasificación propuesta por Heus van Putten y cols. (14) y úlceración. Estos pacientes eran remitidos desde Atención Primaria, Especializada, o desde Hospitalización inmediatamente después de detectada la lesión. En todos ellos se realizó una historia clínica detallada y exploración física completa recogidas en un protocolo de investigación. Los pacientes con lesiones superiores al grado 3 (gangrena) según la clasificación de Wagner fueron excluidos del estudio.

En 14 de los 152 sujetos fue necesaria la amputación como tratamiento de su lesión. En todos los sujetos, antes de la amputación se practicó una arteriografía por sustracción para evaluar el árbol vascular de los miembros inferiores. En un paciente se intentó revascularización por angioplastia y en el resto se utilizaron diferentes técnicas de cirugía vascular con la intención de evitar la amputación .

\section{MÉTODOS}

\section{Clínicos}

Las variables recogidas asociadas con la evolución de las lesiones fueron:

Edad, sexo, tiempo de evolución y tipo de diabetes, antecedentes de úlcera o amputación previas, deformidad o hiperqueratosis, aislamiento social e higiene deficiente de pies.

Grado de retinopatía definido como: grado 1 simple o exudativa, grado 2 preproliferativa, grado 3 proliferativa y grado 4 ceguera. Grado de nefropatía definida como: grado 1: presencia de microalbuminuria en tres muestra de orina de 24 horas positivas (albumina $>30 \mu \mathrm{g} / 24$ horas), grado 2: macroalbuminuria (albumina > $300 \mu \mathrm{g} / 24$ horas) grado 3: insuficiencia renal terminal. Vasculopatía periférica definida: leve por la presencia de claudicación intermitente $>100$ metros con índice tobillo/brazo $<0,9$, y grave por claudicación $<100$ metros con índice tobillo/brazo < 0,6. Grado de neuropatía por criterios clínicos atendiendo a la clasificación "Neurological Disability Score" o NDS (15). Infección grave definida como la persistencia de supuración y celulitis $>2 \mathrm{~cm}$ de diámetro durante una semana con o sin microbiología positiva tras antibióticoterapia empírica. Como antibioticoterapia empírica se utilizó en las ulceraciones grado 1 (Clasificación de Wagner) amoxiclavulánico $500 \mathrm{mg} /$ oral/8 horas, en las grado 2 amoxiclavulánico $500 \mathrm{mg} /$ oral/8 horas o ciprofloxacina 500 $\mathrm{mg} /$ oral/12 horas y clindamicina $1200 \mathrm{mg} /$ oral/día repartida en 4 tomas y grado 3 ciprofloxacina $200 \mathrm{mg} / \mathrm{ev} / 12$ horas y clindamicina $1600 \mathrm{mg} / \mathrm{ev} /$ día.
Se midió el diámetro máximo de la úlcera y se clasificó la lesión atendiendo a los grados de la clasificación de Wagner. En todas las úlceraciones se siguió un mismo protocolo de curas consensuado con el Área Sanitaria, basado en el lavado con agua y jabón ( $\mathrm{pH}$ ácido), suero fisiológico estéril, uso de gasa estéril, recorte de bordes y recovecos con material quirúrgico estéril en la sala de curas, pomadas enzimáticas o cicatrizantes dependiendo de la evolución de las lesiones y pintado del borde con solución yodada.

A los pacientes se les entregó por escrito y se les explicó en todas las consultas los cuidados mínimos del pie.

En todos los afectados se práctico en la primera visita o en sucesivas visitas ante una mala evolución de las lesiones una radiografía simple antero-posterior y lateral del pie.

\section{Bioquímicos}

Tras 8 horas de ayuno se determinó en sangre venosa hemograma, glucemia (método enzimático), HbA1c (cromatografía), creatinina, colesterol total (método enzimático), triglicéridos (método enzimático), HDL colesterol (precipitación con polianiones) y en orina de 24 horas creatinuria y microalbuminuria (tres muestras consecutivas).

\section{Estadísticos}

Los datos se presentan como media + desviación estándar. El análisis estadístico se realizó con el programa informático SSPS. Para la comparación entre medias utilizamos la prueba de Mann Withney. Para la comparación de proporciones la prueba de Chi o en caso de que $\mathrm{n}$ fuera menor de 5 el test de Fisher. Para el análisis de factores independientes utilizamos la regresión logística. Se consideró estadísticamente significativo una probabilidad menor al $5 \%$.

\section{RESULTADOS}

En 14 individuos $(9,2 \%)$ de los 152 estudiados fue necesaria la amputación por la mala evolución de la lesión. En cinco individuos la amputación fue mayor. Las características clínicas y bioquímicas de los sujetos estudiados, así como los factores asociados con amputación (AMP) se presentan en la tabla 1. Los factores asociados con amputación en nuestra muestra fueron: presencia de antecedentes personales de amputación, infección grave, osteomielitis, retinopatía proliferativa y signos de vasculopatía periférica (VP) grave.

No encontramos diferencias estadísticamente significativas entre ambos grupos con respecto a otros parámetros analizados como los niveles de colesterol total, HDLc, triglicéridos ni creatinina (datos no mostrados).

Las odds ratio para la amputación fueron respectivamente: antecedentes de AMP (3.7; 1.0113.7), infección grave (14.4; 2.92-71.2) y signos de vasculopatía periférica grave $(7.1 ; 1.88$ 27.2). El análisis con regresión logística (variable dependiente amputación) mostró como variables independientes de asociación con amputación la presencia de VP grave ( $\mathrm{r} 0,66 \mathrm{p}=$ 0,013 ) e infección grave (r 0,69, p = 0,0046). 
TABLA I

\section{PRINCIPALES CARACTERÍSTICAS CLÍNICO BIO LÓ GICAS Y FACTO RES ASO CIADO S CON AM PUTACIÓN EN LOS 152 DIABÉTICOS ESTUDIADOS}

\begin{tabular}{lccc}
\hline & AM P ( $\mathrm{n}=14)$ & No AMP $(\mathrm{n}=138)$ & $\mathrm{P}$ \\
\hline Edad (años) & $72 \pm 7$ & $68 \pm 12$ & $\mathrm{NS}$ \\
Sexo (H/M) & $53 \% / 47 \%$ & $49 \% / 51 \%$ & $\mathrm{NS}$ \\
Tiempo de evolución & & & \\
$\quad$ (años) & $15 \pm 9$ & $18 \pm 7$ & $\mathrm{NS}$ \\
Tipo diabetes (1/2) & $7 \% / 93 \%$ & $10 \% / 90 \%$ & $\mathrm{NS}$ \\
Antecedentes de & & & \\
$\quad$ amputación & $50 \%$ & $21 \%$ & 0,036 \\
Retinopatía grado 2 & $86 \%$ & $49 \%$ & 0,05 \\
Oseomielitis & $27 \%$ & $10 \%$ & 0,05 \\
IM C (kg/m2) & $26,4 \pm 2,9$ & $29,1 \pm 4,6$ & $\mathrm{NS}$ \\
Diámetro máximo de & & & \\
$\quad$ la úlcera & $3,9 \pm 4,6$ & $2,5 \pm 2,3$ & $\mathrm{NS}$ \\
Infección grave & $80 \%$ & $21 \%$ & $<0,0001$ \\
Vasculopatía periférica & & & \\
$\quad$ grave & $50 \%$ & $12 \%$ & 0,001 \\
Glucemia en ayunas & & & \\
$\quad$ (mg/dl) & $139,3 \pm 41,1$ & $166,3 \pm 63,7$ & $\mathrm{NS}$ \\
HBAlc (\%) & $8,1 \pm 1,5$ & $8,1 \pm 1,7$ & $\mathrm{NS}$
\end{tabular}

Abreviaturas: AM P: amputación no traumática, IMC: índice de masa corporal.

\section{DISCUSIÓN}

Las úlceras en el pie diabético generan un elevado coste para el paciente y la sociedad. Para los pacientes suponen sufrimiento, incapacidad, disminución de la calidad de vida, riesgo de pérdida del miembro afectado y aumento de mortalidad $(17,18)$. Para la sociedad suponen un elevado coste por incapacidad laboral y por la utilización del Sistema de Salud. En Suecia la aparición de gangrena representa un $25 \%$ del coste de la atención hospitalaria del diabético (19) y en un reciente estudio de costes del tratamiento de la úlcera diabética en 274 pacientes, los costes más elevados los presentaron los cuidados hospitalarios y el tratamiento tópico (20).

Datos de Calle y cols. (21) indican que en España la incidencia anual de amputaciones no traumáticas es de 46.1 por 100.000 en diabéticos, siendo en población no diabética del 1.6. Mientras que la incidencia en UK es muy elevada siendo de 248 para población diabética y de 20 para no diabéticos (22).

Por tanto, el conocimiento de factores clínicos que fácilmente puedan ser utilizados en Atención Primaria y en las Unidades de Diabetes, y que permitan identificar pacientes de alto riesgo para desarrollar amputación son muy importantes. Una clasificación precoz de riesgo para amputación permite dirigir programas específicos de educación, prevención de lesiones en pie y tratamiento evitando costosos ingresos hospitalarios y amputaciones (23).
En nuestro estudio los factores asociados con amputación en diabéticos de alto riesgo que presentaban ulceración son de forma independiente la presencia infección grave (persistencia de supuración y celulitis $>2 \mathrm{~cm}$ de diámetro durante una semana tras antibióticoterapia empírica), y la vasculopatía periférica grave (claudicación intermitente $<100$ e índice tobillo/brazo $<0,6$ ), siendo dependientes los antecedentes de amputación, el desarrollo de osteomielitis y la retinopatía proliferativa.

Desde estudios clásicos que tratan de identificar factores de riesgo se conoce la importancia de los antecedentes de amputación (24), dado que la probabilidad en un año de que un diabético presente una nueva amputación en el miembro contralateral es de hasta un $30 \%$ (25). Los bajos índices tobillo/brazo y el grado de claudicación intermitente es un marcador clínico de vasculopatía avanzada. En nuestro estudio la presencia de vasculopatía avanzada da un riesgo 7 veces mayor para amputación, este factor también ha sido investigado por otros autores asociándolo a elevado riesgo y traduce la experiencia clínica de que en las úlceraciones con pulso presente el porcentaje de amputaciones es mínimo. Así, Yeager y cols. (26) demostraron en 162 sujetos (72\% DM) recogidos desde 1992 - 1996 que necesitaron AMP menor y que fueron seguidos durante 25 meses que el predictor de no curación y AMP mayor fue la falta de revascularización. En cambio, los pacientes con pulso palpable y úlceras neuropáticas necesitaron cirugía pero no AMP mayor. Lehto y cols., siguiendo a 1044 DM tipo 2 durante 7 años demostraron que los predictores de AMP fueron, además de niveles elevados de glucemia basal y HbA1c (con efecto dosis respuesta), los signos de VP (ausencia de pulsos) y signos de neuropatía avanzada (ausencia de ROT y sensibilidad vibratoria) (27).

La mala evolución de la infección al no ser adecuadamente controlada por la antibiticoterapia empírica hace que el riesgo de amputación se multiplique 14 veces. Este dato indica la importancia del estrecho seguimiento en la evolución clínica de las lesiones durante la primera semana y obliga a citar diariamente a los pacientes para realizar las curas y el seguimiento clínico. También indica la necesidad de instaurar una antibioticoterapia empírica agresiva ante lesiones agudas del pie diabético. Este factor de infección no controlada también aparece como independiente en el estudio de Morris y cols. (22).

Otros estudios muestran una asociación y predicción de AMP con otros factores como el control metabólico (27), duración de la diabetes (28), mala educación diabetológica y falta de cuidado en los pies $(28,29)$ y los signos de neuropatía avanzada $(25,27)$.

En nuestro estudio probablemente no encontramos relación con la duración de la diabetes porque se trata preferentemente de diabéticos tipo 2 en los que es difícil establecer el comienzo exacto de la enfermedad, siendo gran número de casos no diagnósticados hasta que aparecen las complicaciones clínicas. Tampoco encontramos relación con el control metabólico dado que nuestro estudio es transversal y sólo recoge la HbA1c a la entrada en la Unidad del Pie diabético, lo que no refleja el control metabólico previo de los pacientes. No encontramos asociación con falta de cuidado en pies pero si con la retinopatía avanzada que indica la imposibilidad de una correcta autoexploración y cuidados del pie por parte del paciente. Este factor junto con la incapacidad motora también es muy importante en el estudio de Calle y cols. (21). Tampoco encontramos una asociación con el grado de neuropatía 
periférica dado que era un criterio de selección para entrar en el estudio (clasificación propuesta por Heus van Putten MA (14).

En conclusión, los pacientes diabéticos con lesiones agudas en pie que presenten antecedentes de amputación, signos clínicos de vasculopatía periférica, infección grave que no evoluciona correctamente con antibiótico-terapia empírica, evolución hacia la osteomielitis o con retinopatía avanzada son de alto riesgo para la amputación y en ellos se deben instaurar precozmente medidas terapéuticas y preventivas intensas.

\section{Bibliografía}

1. Centers for Disease Control and Prevention, Atlanta GA, 1997.

2. American Diabetes Association: Diabetes: 1991 Vital Statistics. Alexandria, VA American Diabetes Association, 1991.

3. Freedman DS, Gruchow HW, Bamrah VS, Anderson AJ, Barboria KJJ. Diabetes mellitus and arteriographically documented coronary artery disease. J Clin Epidemiol 1988; 41: 659-68.

4. Huse DM, Oster G, Killen AR, Lacey MJ, Colditz GA. The economic costs of non-insulin-depent diabetes mellitus. JAMA 1989; 262: 2708-13.

5. Rubin RJ, Altman WM, Mendelson DN. Health care expenditures for people with diabetes mellitus. J Clin Endocrinol Metab 1992; 78: 801-9.

6. Nathan DM. Long-term complications of diabetes mellitus. N Engl Med 1993; 328: 1676-83.

7. Hiller R, Sperduto RD, Podgor MJ, Ferris FL, Wilson PWF. Diabetic retinopathy and cardiovascular disease in type 11 diabetics: the Framingham Heart Study and the Framingham Eye Study. Am J Epidemiol 1988; 128: 402-10.

8. Most RS, Sinnock P. The epidemiology of lower extremity amputations in diabetic individuals. Diabetes Care 1983; 6: 87-90.

9. American Diabetes Association. Report from the American Diabetes Association Economic consequences of diabetes mellitus in the US in 1997. Diabetes Care 1998; 21: 296-309.

10. Warren M, Espinosa C, Rovira J. El coste de la diabetes mellitus conocida en España. Med Clin (Barc) 1997; 109: 289-93.

11. Reiber GE. The Epidemiology of diabetic foot problems. Diabetic Med 1996; 13: S6-S11.

12. Bouter KP, Storm AJ, de Groot RRM, Uitslager R, Erkelens DW, Diepersloot RJA. The diabetic foot in Ducht hospitals: epidemiological features and clinical outcome Eur J Med 1993; 2: 215-8.

13. Sims DC, Cavanagh PR, Ulbrecht JS. Risk factors in the diabetic foot. Recognition and management. Physical Therapy 1988; 68: 1887-1902.

14. Heus van Putten MA, Schaper NC, Bakker K. The clinical examination of the diabetic foot in daily practise. Diabetic Medicine 1996; 13: S5558.

15. Young MJ, Boulton AJM, McLeod AF, Williams DRR, Sonske PH. A multicentric study of the prevalence of diabetic peripheral neuropathy in the United Kingdom hospital clinic population. Diabetologia 1993; 36: 150-4.

16. Levin ME. Preventing amputation in the patient with diabetes. Diabetes Care 1995; 18: 1383-5.

17. Pecoraro RE. The non-healing diabetic ulcer, a major cause for limb loss. Prog Clin Biol Res 1991; 365: 27-43.

18. Reiber GE. Diabetic foot care. Diabetes Care 1992; 15: 29-31.

19. Jonson B. Diabetes the cost of illness and the cost of control. Acta Med Scan 1983; 671: 463-71.

20. Apelqvist J, Castenfors J, Larsson J, Stenstrom A, Agardh CD. Diabetes foot ulcer in a multidisciplinary setting. An economic analysis of primary healing and healing with amputation. J Int Med 1994; 235 : 453-71.

21. Calle-Pascual AL, Redondo MJ, Ballesteros M, Martinez-Salinas MA, Diaz JA, De Matias P, et al. Nontraumatic lower extremity amputations in diabetic and non diabetic subjects in Madrid, Spain. Diabetes \& Metabolism (Paris) 1997; 23: 519-23.

22. Morris AD, Mc Alpine R, Steinke D, Boyle Dl, Ebrahim AR, Vasudev $\mathrm{N}$, et al. Diabetes and lower limb amputation in the community. A retrospective cohort study DARTS/MEMO collaboration. Diabetes audit and research in Tayside Scotland/Medicines monitoring unit. Diabetes Care 1998; 21: 738-43.

23. Rith Najarian S, Braunchaud C, Beaulieu O, Gohdes D, Simonson G, Mazze R. Reducing lower extremity amputations due to diabetes. Application of the staged diabetes management approach in a primary care setting. J Fam Pract 1998; 47: 127-32.

24. Mayfield JA, Reiber GE, Nelson RG, Greene T. A foot risk classification system to predict amputation in Pima Indians. Diabetes Care. 1996;19: 704-9.

25. Widmer L. Course of occlusive peripheral artery disease in early detected patients . Basel study 11 years follow up. En Maurer PC ed. Whats new in angiology?. Munich Strano ed, 1986; 13-5.

26. Yeager RA, Moneta GL, Edwards JM, Williamson WK, McConnell DB, Taylor LM, Porter JM. Predictors of outcome of forefoot surgery for ulceration and gangrene. Am J Surg 1998; 175: 388-90.

27. Lehto S, Ronnemaa T, Pyorala, K, Laakso M. Risk factors predicting lower extremity amputation in patients with NIDDM. Diabetes Care 1996; 19: 607-12

28. Flores-Rivera AR. Risk factors for lower amputation in diabetic patients: a case- control study. Arch Med Res 1998; 29: 179-84.

29. Humphrey AR, Dowse GK, Thoma K, Zimmet PZ. Diabetes and non traumatic lower extremity amputations. Incidence, risk factors and prevention a 12 year follow up study in Naru. Diabetes Care 1996; 19: 710-4. 